Pacific

Journal of

Mathematics

DIMENSION REDUCTION UNDER THE RICCI FLOW ON MANIFOLDS WITH NONNEGATIVE CURVATURE OPERATOR

XiaOdong CAO

Volume $232 \quad$ No. 2

October 2007 


\title{
DIMENSION REDUCTION UNDER THE RICCI FLOW ON MANIFOLDS WITH NONNEGATIVE CURVATURE OPERATOR
}

\author{
XiaOdOng CAO
}

\begin{abstract}
In this paper, we study the dilation limit of solutions to the Ricci flow on manifolds with nonnegative curvature operator. We first show that such a dilation limit must be a product of a compact ancient Type I solution of the Ricci flow with flat factors. Then we show that, under the Type I normalized Ricci flow, the compact factor has a subsequence converging to a Ricci soliton.
\end{abstract}

\section{Introduction}

Let $M$ be a complete Riemannian manifold. A maximal solution to the Ricci flow

$$
\frac{\partial}{\partial t} g_{i j}=-2 R_{i j}
$$

on $M$ is a time-dependent family of complete Riemannian metrics $g(t) . M$ is either compact or complete noncompact with bounded curvature at each time $t$, and we assume that the Riemannian curvature $|R m|$ becomes unbounded at some finite time $T$. Furthermore, we assume that the curvature operator of the initial metric is nonnegative. It is well known that the nonnegativity is preserved by the Ricci flow.

One way to analyze the singularities for the Ricci flow is to study singularity models. The basic idea is to blow up maximal solutions around points of maximum curvature, reparameterize in both space and time, and then take a limit. We can classify all the singularity models into three types according to their curvature growth: ancient, eternal and immortal solutions. Even if the original manifold is compact, the limit can be complete noncompact.

The study of singular solutions is a main topic in the Ricci flow and other geometric evolution equations. In particular, analyzing the singularity models gives important information about the geometric and topological structure of the manifold.

Dimension reduction is a useful tool for studying the singularities of minimal surface theory and harmonic map theory. The main idea is that, after having taken

MSC2000: 53C44.

Keywords: Ricci flow. 
a limit of a sequence of dilations to get a singularity model, one can take another sequence of origins going out to infinity and shrinking back down to get a new limit of lower dimension. Hamilton [1995b] brought this idea to study singularities of the Ricci flow on manifolds with nonnegative curvature operator, and he used this idea to study the singularity formation of the Ricci flow. H.-D. Cao [2004] used a similar idea to study the formation of singularities of the Kähler-Ricci flow in noncompact complex dimension $n=2$ and with bounded nonnegative bisectional curvature.

There is one important geometric invariant that is very useful in the theory of dimension reduction in Ricci flow. On a complete noncompact Riemannian manifold, let $\mathrm{O}$ be an origin, $s=s(x)$ be the distance to the origin, and $R(x)$ be the scalar curvature. One can define the asymptotic scalar curvature ratio (or ASCR) by

$$
\operatorname{ASCR}(g)=\limsup _{s \rightarrow \infty} R(x) s^{2} .
$$

It is well known that this definition is independent of the choice of origin and invariant under dilation. Also, on a complete ancient solution with bounded nonnegative curvature operator, $\operatorname{ASCR}(g(t))$ is a constant in time $t$.

Hamilton [1995b] first pointed out that if $\operatorname{ASCR}(g(t))=\infty$, then one can choose a sequence of points going out to infinity, perform dimension reduction, and hence get a limit that splits as the product of a lower dimensional manifold with flat factors. More details can be found in the wonderful book [Chow et al. 2006].

We begin the rest of this paper with Section 2, where we will first refine Hamilton's dimension reduction theorem and then state our main theorems. In Section 3, we will detail the Type I solution to the Ricci flow and its normalization. Then, in Sections 4 and 5, we will prove the subconvergence to a Ricci soliton.

\section{Dimension reduction}

Let us first recall a dimension reduction theorem:

Theorem 1 [Hamilton 1995b]. Suppose we have a solution to the Ricci flow on a compact manifold $M^{m}$ of dimension $m$ with weakly positive curvature operator for a maximal time interval $0 \leq t<T$; then we can find a sequence of dilations that converge to a complete solution of the Ricci flow with curvature bounded on an ancient time interval $-\infty<t<\Omega$. The solution has scalar curvature bounded by

$$
R \leq \Omega /(\Omega-t)
$$

everywhere and has $R=1$ at some origin $O$ at time $t=0$; the limiting solution again has weakly positive curvature operator. Moreover the limit splits as a quotient of a product $N^{n} \times R^{k}$ with $m=n+k$ flat in the direction $R^{k}$ with $k \geq 0$, where 
the interesting factor $N^{n}$ either is compact or has finite asymptotic curvature ratio

$$
\mathrm{ASCR}=\lim _{s \rightarrow \infty} R s^{2}<\infty .
$$

Moreover the limit factor $N^{n}$ will still satisfy a local injectivity radius estimate.

Note that, in the above theorem, $\Omega$ could be infinity. The injectivity radius estimate now follows from the work of Perelman [2002].

Our first theorem is:

Theorem 2. In Theorem 1, where $k$ is maximum, $N^{n}$ must be a compact and Type I solution to the Ricci flow with nonnegative curvature operator. Moreover, the diameter is bounded by $c / \sqrt{R_{\max }}$ for some constant $c$.

When the solution is Type I, the Ricci flow normalization differs from the standard one, which preserves the volume. We can dilate the space so that the maximum curvature is always bounded.

Our second theorem says that the above $N^{n}$ has a subsequence converging to a gradient Ricci soliton under the Type I normalized Ricci flow:

$$
\frac{\partial}{\partial t} g_{i j}=-2 R_{i j}+g_{i j}
$$

Theorem 3. Let $\left(N^{n}, g(t)\right)$ be obtained as above. Then, under the Type I normalized Ricci flow, the solution exists and the curvature is bounded for all time. Also, as $t \rightarrow \infty$, the solution has a subsequence converging to a gradient Ricci soliton.

In other words, those dilation limits look like a product of a shrinking Ricci soliton and $R^{k}$.

\section{Type I solution}

Here we detail the Type I solution to the Ricci flow. Consider a maximal solution $(M, g(t))$ to the Ricci flow on a manifold $M$ for $0 \leq t<T$, where either $M$ is compact or at each time $t$ the metric $g(t)$ is complete with bounded curvature, and either $T=\infty$ or $|R m|$ becomes unbounded as $t \rightarrow T$. Let $M(t)$ denote the maximal curvature at time $t$ :

$$
M(t)=\sup _{x \in M}\{|R m(x, t)|\} .
$$

We say a maximal solution is Type I if $T<\infty$ and

$$
\sup (T-t) M(t)<\infty .
$$

If we assume that the curvature operator is nonnegative, we can then replace the Riemannian curvature by the scalar curvature in the above, that is,

$$
M(t)=\sup _{x \in M} R(x, t) .
$$


So for a Type I solution, we have

$$
|R m(x, t)| \leq \frac{C}{T-t} \quad \text { for some constant } C .
$$

In studying the Ricci flow, it is sometimes easier to study normalized flows. For example, Hamilton [1982; 1999] considers the Ricci flow normalized to preserve the volume. The unnormalized and normalized Ricci flows differ only in spacial scale and by a parameterization in time. For a Type I solution, we can consider a different normalization, but one that also only differs in space and time scales. Here we rescale space by a factor of $1 / \sqrt{M(t)}$ so that, after rescaling, the maximum curvature is always bounded by some constant $C$.

More precisely, we let

$$
\tilde{t}=\int_{0}^{t} \frac{1}{T-z} d z=-\ln (T-t)+\ln T \quad \text { and } \quad \tilde{g}(\tilde{t})=\frac{1}{T-t} g(t) .
$$

Hence the curvature now satisfies

$$
|\tilde{R m}(x, \tilde{t})|=(T-t)|\operatorname{Rm}(x, t)| \leq C .
$$

It is easy to verify that $(M, \tilde{g}(\tilde{t}))$ now satisfies the evolution equation

$$
\frac{\partial}{\partial \tilde{t}} \tilde{g}_{i j}=\frac{\partial}{\partial t} \tilde{g} \cdot \frac{\partial t}{\partial \tilde{t}}=-2 \tilde{R}_{i j}+\tilde{g}_{i j}
$$

We call this Type I normalized Ricci flow. N. Sesum told us later that this flow is same as her $\tau$-flow when $\tau=1$. By applying Hamilton's compactness theorem [1995a], we have:

Theorem 4. Let $(M, g(t))$ be a Type I solution of the Ricci flow, where $M$ is a compact Riemannian manifold. Then, under the Type I normalized Ricci flow, the solution exists and the curvature is bounded for all time. As $t \rightarrow \infty$, the solution has a subsequence converging to a (possibly complete) Riemannian manifold $(N, h(t))$, which is also a solution to the Type I normalized Ricci flow.

Remark 5. The injectivity radius bound follows from [Perelman 2002].

\section{Proof of Theorem 2}

Before we start proving our theorem, we need another result.

Proposition 6 [Perelman 2002]. A complete nonflat ancient solution with bounded curvature and nonnegative curvature operator has asymptotic volume ratio $v=0$ and asymptotic scalar curvature ratio $\mathrm{ASCR}=\infty$.

Proof of Theorem 2. We first notice that in Theorem 1, $N^{n}$ (compact or complete with $A<\infty$ ) must be an ancient solution to the Ricci flow, and it must be Type 
I. Since a Type II limit with weakly positive curvature operator must be a Ricci soliton [Hamilton 1995b], it must have ASCR $=\infty$.

Now we claim that $N^{n}$ can not be complete noncompact. Note that if $N^{n}$ is a complete ancient solution with bounded curvature and nonnegative curvature operator, which is obtained from Theorem 1, then we will have ASCR $<\infty$. This contradicts Proposition 6. So $N^{n}$ must be compact and Type I.

Next we show that $N^{n}$ must satisfy for as long as the solution exists that

$$
\operatorname{diam}(N, g(t)) \leq \frac{c}{\sqrt{R_{\max }}},
$$

for some universal constant $c$.

Otherwise we can always try to dilate $N^{n}$ again by $R_{\max }$ at each time and take another limit. Then either we will get a complete limit with ASCR $=\infty$, and hence we can perform another dimension reduction to get at least another flat factor $R^{1}$. This which will contradict that $k$ is maximum. Or, we will get a complete limit with ASCR $<\infty$, which contradicts Proposition 6 . That means the only possible limit $N^{n}$ is a compact Type I, ancient solution to the Ricci flow with bounded diameter comparable to maximum curvature at any time.

\section{Proof of Theorem 3}

In this section, we show that the solution

$$
\frac{\partial}{\partial \tilde{t}} \tilde{g}_{i j}=-2 \tilde{R}_{i j}+\tilde{g}_{i j}
$$

of the Type I normalized Ricci flow exists for all time and has a subsequence converging to a Ricci soliton. Note for our solution $(N, g(t))$ to the Ricci flow, we have a nonnegative curvature operator and a scalar curvature that satisfies

$$
R \leq \frac{C}{T-t}
$$

under the Ricci flow. The Type I normalized Ricci flow is a scaling of the Ricci flow, with scaling factor $1 / T-t$. So our solution to the Type I normalized Ricci flow will also have a nonnegative curvature operator and a scalar curvature satisfying

$$
\tilde{R} \leq C,
$$

Since

$$
\tilde{t}=-\ln (T-t)+\ln T,
$$

we have $\tilde{t} \rightarrow \infty$ as $t \rightarrow T$.

The diameter of $(N, \tilde{g}(t))$ now satisfies

$$
\operatorname{diam}(N, \tilde{g}(t)) \leq c .
$$


The subsequence's convergence now follows similarly as in the proof of [Sesum 2004], which says that if the solution to the $\tau$-flow exists for all time and the curvature and diameter are bounded, then there exists a subsequence that converges to a Ricci soliton. In fact, the $\tau$-flow and the Type I normalized Ricci flow differ only by a constant factor.

\section{Acknowledgement}

The author thanks Professor Ben Chow for many valuable discussions of work and Professors Richard Hamilton and Gang Tian for their encouragement, constant support, and advice. He thanks Professor Duong H. Phong for his interest and helpful suggestions. He also thanks the Mathematical Sciences Research Institute and Clay Mathematics Institute, because part of this work was carried out when he was visiting the Clay summer program at MSRI in 2005.

\section{References}

[Cao 2004] H.-D. Cao, "On dimension reduction in the Kähler-Ricci flow", Comm. Anal. Geom. 12:1-2 (2004), 305-320. MR 2005d:53104 Zbl 1075.53058

[Chow et al. 2006] B. Chow, P. Lu, and L. Ni, Hamilton's Ricci flow, Graduate Studies in Mathematics 77, American Mathematical Society, Providence, RI, 2006. MR 2274812 Zbl 1118.53001

[Hamilton 1982] R. S. Hamilton, "Three-manifolds with positive Ricci curvature", J. Differential Geom. 17:2 (1982), 255-306. MR 84a:53050 Zbl 0504.53034

[Hamilton 1995a] R. S. Hamilton, "A compactness property for solutions of the Ricci flow", Amer. J. Math. 117:3 (1995), 545-572. MR 96c:53056 Zbl 0840.53029

[Hamilton 1995b] R. S. Hamilton, "The formation of singularities in the Ricci flow", pp. 7-136 in Surveys in differential geometry (Cambridge, MA, 1993), vol. II, edited by C. C. Hsiung and S. T. Yau, International Press, Cambridge, MA, 1995. MR 97e:53075 Zbl 0867.53030

[Hamilton 1999] R. S. Hamilton, "Non-singular solutions of the Ricci flow on three-manifolds", Comm. Anal. Geom. 7:4 (1999), 695-729. MR 2000g:53034 Zbl 0939.53024

[Perelman 2002] G. Perelman, "The entropy formula for the Ricci flow and its geometric applications", Preprint, 2002, Available at http://arxiv.org/abs/math/0211159.

[Sesum 2004] N. Sesum, "Limiting behaviour of the Ricci flow", Preprint, 2004, Available at http:// arxiv.org/abs/math/0402194.

Received July 4, 2006. Revised December 2, 2006.

Xiaodong CAO

DEPARTMENT OF MATHEMATiCs

507 MALOTT HALL

CORNELL UNIVERSITY

ITHACA, NY 14853-4201

UNITED STATES

cao@math.cornell.edu

http://www.math.cornell.edu/People/Faculty/caox.html 